\title{
Video Loss Recovery With FEC and Stream Replication
}

\author{
S.-H. Gary Chan, Senior Member, IEEE, Xing Zheng, Qian Zhang, Senior Member, IEEE, \\ Wen-Wu Zhu, Senior Member, IEEE, and Ya-Qin Zhang, Fellow, IEEE
}

\begin{abstract}
Packet loss is inevitable in video multicast. In this paper, we propose and study an effective feedback-free loss recovery scheme for layered video which combines forward error correction (FEC) and stream replication. In our scheme, the server multicasts the video in parallel with FEC packets and a number of replicated delayed (ReD) version of the stream. Receivers autonomously and dynamically join the FEC and ReD streams to repair their losses. On the server side, we analyze and optimize the number of replicated streams and FEC packets to meet a certain residual loss requirement (i.e., error after correction). On the receiver side, we analyze the optimal combination of FEC and $\operatorname{ReD}$ packets to minimize its loss. We also present a fast yet accurate approximation algorithm for receiver to make such decision. We show that FEC combined with merely one or two replicated streams can effectively reduce the residual error rate (by as much as 50\%) as compared with pure FEC or replication alone. Both subjective and objective video measures confirm that our recovery scheme achieves much better visual quality.
\end{abstract}

Index Terms-Fast approximation, feedback-free error recovery, forward error correction (FEC), layered video multicast, stream replication.

\section{INTRODUCTION}

$\mathbf{M}$ ANY multimedia applications involve point to multipoint communication, i.e., a video sequence stored or generated at a server is simultaneously delivered to a group of receivers distributed in a network [1]. ${ }^{1}$ In multicasting video over networks, packet loss is inevitable. In order to offer goodquality video, it is important to recover most of the loss so that the resultant end-to-end error rate after correction, i.e., the residual loss rate, is kept below a certain value [2]-[4].

Automatic repeat request (ARQ) is clearly not suitable for real-time multicast applications due to recovery delay and implosion problems [5], [6]. Therefore, Forward Error Correction

Manuscript received July 9, 2003; revised April 24, 2005. This work was supported in part by the Areas of Excellence (AoE) Scheme on Information Technology funded by the University Grant Council in Hong Kong (AoE/E01/99), and by the Research Grant Council in Hong Kong (HKUST6199/02E \& HKUST6156/03E). The associate editor coordinating the review of this manuscript and approving it for publication was Dr. Minoru Etoh.

S.-H. G. Chan and Y.-Q. Zhang are with the Department of Computer Science, The Hong Kong University of Science and Technology, Clear Water Bay, Kowloon, Hong Kong (e-mail: gchan@ cs.ust.hk).

X. Zheng was with the Department of Computer Science, The Hong Kong University of Science and Technology, Clear Water Bay, Kowloon, Hong Kong. She is now with the School of Information and Computer Science, University of California, Irvine, CA 92717 USA.

W.-W. Zhu is with the Intel Communication Technology China Lab, Beijing 100080, China (e-mail: wenwu.zhu@intel.com).

Y.-Q. Zhang is with Microsoft Research Asia, Beijing 100080, China.

Digital Object Identifier 10.1109/TMM.2005.864340

${ }^{1}$ In this paper, we use "client" and "receiver" interchangeably.
(FEC) such as the Reed-Solomon correction code is often used. In traditional FEC, for every $K$ source packets, the server introduces $N-K$ parity packets to make up a codeword of $N$ packets. So long as a client receives at least $K$ out of the $N$ packets, it can recover all the $K$ source packets.

FEC, though feedback-free, suffers from a weakness: the number of lost source packets has to be compensated by the receipt of at least the same number of parity packets in order to recover all of the source packets; otherwise, all the parity packets are received in vain. This aggressive "all-or-none" error recovery strategy adversely limits the usefulness of FEC when the loss is (momentarily) high.

To overcome the above weakness, we propose the use of replicated and delayed $(\mathrm{ReD})$ streams to combine with FEC. In our scheme, the server replicates a stream and multicasts them in a delayed manner in parallel with the FEC packets. Since the ReD packets are simply the source packets, they are used to first incrementally recover some of the lost packets. In this way, FEC can be applied more effectively to repair the remaining lost ones. The receivers, depending on their local losses, autonomously joins the recovery streams consisting of the FEC and ReD streams so as to minimize its residual loss rate.

In Fig. 1, we show the receiver-driven multicast system under consideration. The server encodes the video stream into multiple layers [7], [8]. FEC and ReD packets are introduced to each layer as recovery streams. All these layers and recovery streams are multicast in parallel to different groups. The loss probability of the clients may vary over time and widely (from as low as nearly zero to as high as tens of percent). In addition, the receivers may have heterogeneous end-to-end bandwidth which may vary over time. Depending on its local bandwidth, loss probability and loss pattern, a client autonomously chooses the appropriate mix of FEC and ReD packets to maximize its video quality.

We consider that both FEC and ReD packets may also be lost. Given a certain receiver bandwidth, there is an optimal combination of FEC and ReD packets to minimize loss. Suppose a receiver loses a certain number $l$ of source packets. If it gets only FEC packets, it has the risk that if the resultant number of correctly-received FEC packets is less than $l$, all the FEC packets received are useless. On the other hand, if it gets only ReD packets, due to random packet loss, it may end up receiving duplicate copies of the same lost packet. Since multiple copies of a packet are as good as a single one, the recovery is not effective. There is hence an optimal mix of FEC and $\mathrm{ReD}$ packets. 


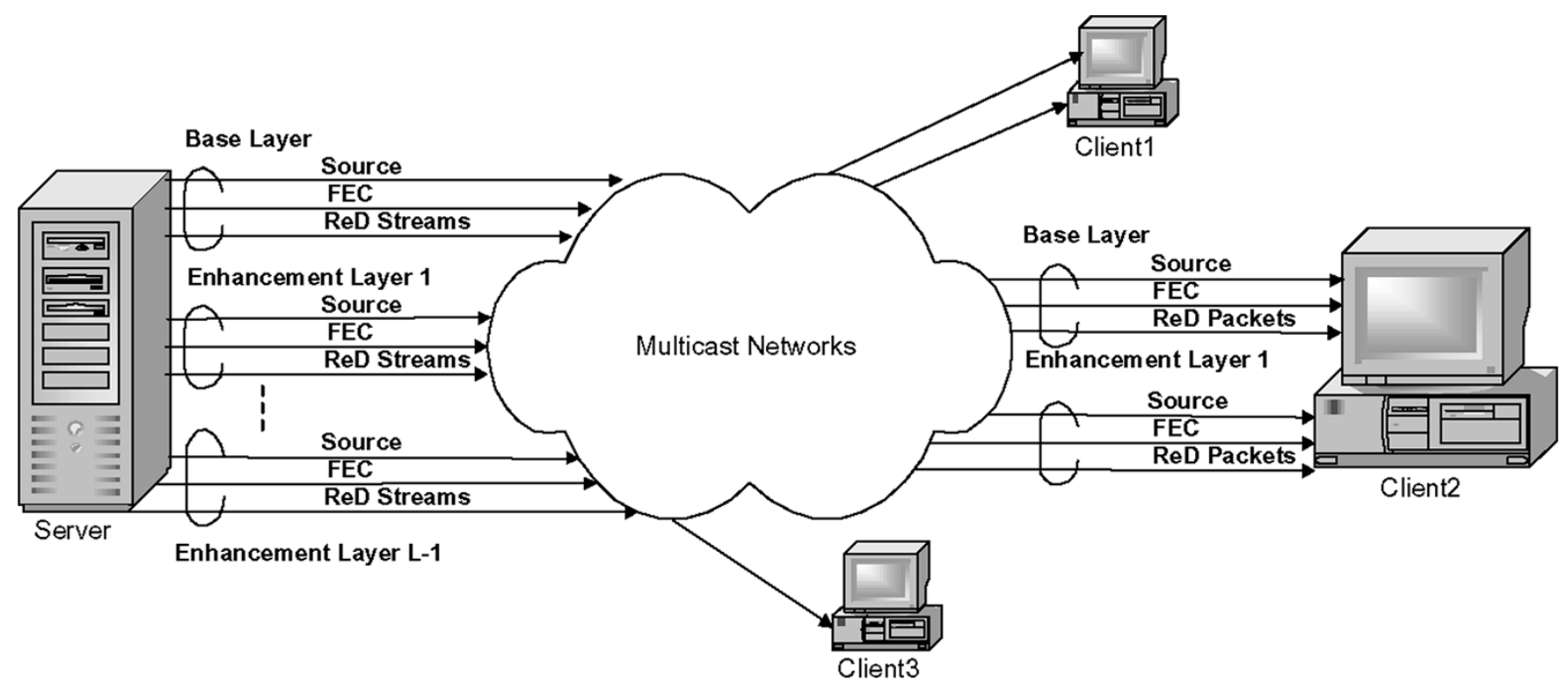

Fig. 1. Layered video multicast with feedback-free error recovery.

TABLE I

Residual ERror RATE FOR PURE FEC, Pure ReD AND OUR HYBRID SCHEMES $(K=30, p=0.2$, and $N=90)$

\begin{tabular}{cr}
\hline Scheme & Residual error rate (\%) \\
\hline FEC & 4.8272 \\
ReD & 2.1047 \\
Hybrid & 1.4838 \\
\hline
\end{tabular}

The residual loss rate can be markedly reduced using our hybrid scheme. As a simple illustration, consider a server which, for every $K=30$ source packets, sends out $N-K=60$ recovery packets. A receiver has enough bandwidth to get at most ten recovery packets for every $K$ source packets. It is experiencing a (momentary) loss rate of $p=0.2$, with the source and recovery packets lost with the same probability. Based on analysis (discussed later in Section III), we show in Table I the residual loss rate for the receiver using pure FEC (i.e., 60 FEC packets for every 30 source packets), pure $\mathrm{ReD}$ (i.e., two replicated streams), and our hybrid scheme ( 30 FEC packets and one replicated stream). Our hybrid scheme clearly out-performs all the other schemes.

We hence address the following three important issues in the system.

1) Server Transmission: The server is designed with a certain target receiver in mind which experiences a typical high (maximum) packet loss rate. It has to decide the number of FEC packets and ReD streams for each layer so as to meet a certain residual error rate requirement (say, 2-5\%) for this receiver.

2) Receiver Selection: The receiver has to decide the optimal combination of FEC and ReD packets in order to minimize its residual error rate, given its local loss pattern and probability, and the server transmission "menu."

3) Fast Allocation Algorithm at the Receiver: Given that network conditions are dynamic and some clients may have low processing capabilities, we devise fast and simple algorithms and closed-form expressions for the receiver to determine the optimal composition of FEC and ReD packets for each layer in order to meet a certain residual error requirement.

We briefly discuss previous work as follows. In multicast, ARQ and FEC are usually studied in the context of providing reliable service [9]-[15]. Since meeting a certain delay constraint is not a major concern in these works, they cannot be directly applied to real-time video multicast with a certain delivery deadline as we consider here. Our work uses ARQ in the form of $\mathrm{ReD}$ packet to eliminate feedback and hence implosion problem. Some other work focuses on building logical repair trees for retransmission so as to efficiently recover errors [16], [17]. Our scheme, as opposed to them, is feedback-free with the use of ReD and FEC packets. Our work makes use of layered FEC as discussed in [18], [19], but differs by addressing the optimal combination of FEC and ReD packets. Applying FEC in wireless channel has been explored in [20]-[22]. However, all of them are based on pure FEC instead of the hybrid scheme we propose here. Combining FEC and ARQ has shown to be effective in [23]-[28]. However, these schemes are studied in the context of reliable multicast, where the objective is to minimize the number of packets sent. We differ by addressing how to effectively recover loss given a certain deadline constraint. An orthogonal work on how to handle network congestion due to stream replication has been discussed in [29]. This can be combined with ours to achieve better recovery. We extend the work in [30] by presenting fast allocation algorithms here. There has been much work on unequal error protection for video transmission (see, for examples, [31]-[38]). The work focuses on how to better protect video sequence against loss through various coding techniques. The UEP codes are usually embedded into the video stream and transmitted together in a single channel. As opposed to this, we consider multiple recovery channels which a receiver may dynamically join to effectively repair their lost packets. Our scheme is orthogonal and complementary to them, 


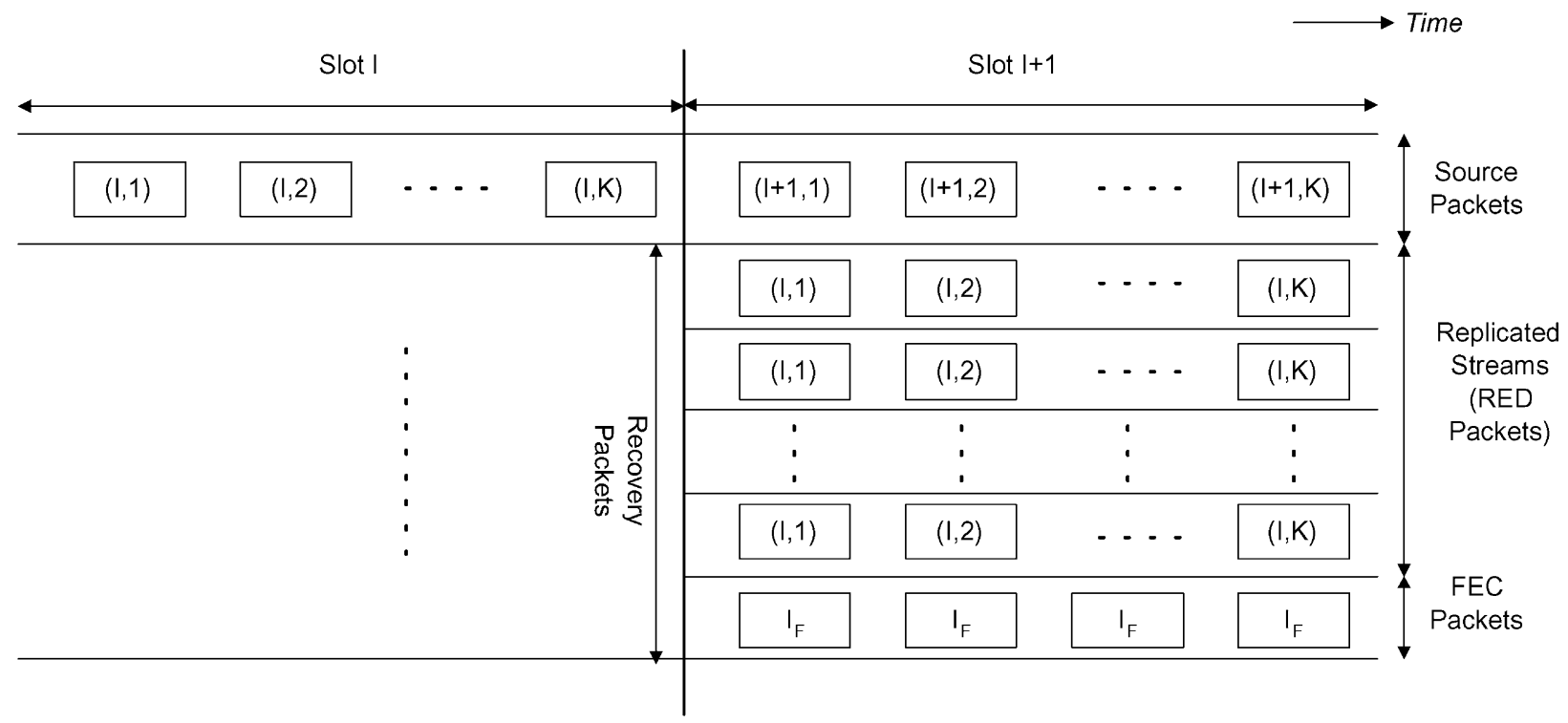

Fig. 2. Server's menu.

and can be applied in conjunction with UEP to further reduce residual error rate.

This paper is organized as follows. In Section II, we describe the system and its problem formulation. The analysis on residual error rate is then presented in Section III. In Section IV, we present the fast allocation algorithm at the receiver. We show in Section V some illustrative numerical results. We finally conclude in Section VI.

\section{SySTEM OPERATION}

In this section, we describe the system in regards to server and receiver operations and formulate the problems. Without loss of generality, we focus in this section on a particular video layer of a certain bandwidth. The issue of multiple layers and the joint allocation of bandwidth, FEC, and ReD packets for each layer will be discussed in Section IV.

\section{A. Server Operation}

The time is divided into slots of fixed length. In each slot, the server multicasts $K$ fixed-size source packets and $N-K$ recovery packets. We show the server's transmission schedule at time slots $I$ and $I+1$ in Fig. 2 . At time slot $I$, the server sends $K$ source packets labeled as $(I, 1),(I, 2), \ldots,(I, K)$. The recovery packets corresponding to the block are sent in the following time slot $I+1$ in parallel as shown. If the layer is replicated $M$ times, all the $M \operatorname{ReD}$ streams are transmitted using different groups with a total of

$$
N_{R} \equiv M \cdot K
$$

ReD packets. There are also $N_{F}$ FEC packets transmitted in another multicast group, where

$$
N_{R}+N_{F}+K=N
$$

We call $\left(N_{F}, M\right)$ the menu of the server. A receiver may join the multicast groups to get the necessary recovery packets at the beginning of slot $I+1$ upon discovering errors at the end of slot
I. (Such join and leave mechanisms to get recovery packets has also been used in [39]).

\section{B. Receiver Operation}

A receiver periodically estimates its loss rate and bandwidth in each slot [40]-[43]. Let $n$ be the receiver's bandwidth in terms of the maximum number of (correct or not) packets it can receive in one time slot. In other words, the receiver can subscribe at most $n-K$ recovery packets in a slot $(n \geq K)$. We further let $p$ be the loss probability of a packet in a slot, independent identically distributed over all packets in the slot. Note that $p$ is the spot rate and hence may vary from slot to slot. For the sake of simplicity, we will assume that both $p$ and $n$ are constant over time in this paper even though our system does not require them to be so.

At each slot, a receiver selectively joins a subset of the recovery streams, i.e., the ReD and FEC streams, depending on its loss condition. More concretely, out of the $K$ source packets the receiver subscribes in time slot $I$, a random number, say $l$, of the packets are lost $(0 \leq l \leq K)$. The recovery is done at slot boundaries according to the following steps.

1) A receiver always joins the stream with the source packets.

2) At the end of slot $I$, the receiver knows $l$ and makes a decision on how many FEC and ReD packets to get in slot $I+1$. Let $n_{F}$ and $n_{R}$ be the number of FEC and ReD packets to get, respectively. Note that both $n_{F}$ and $n_{R}$ are random numbers and functions of $l$.

3) At the beginning of slot $I+1$, the receiver selectively joins the respective recovery streams for durations enough to get $n_{F}$ FEC and $n_{R}$ ReD packets. Note that some of these FEC and ReD packets may be received in error.

4) At the end of slot $I+1$, the receiver performs error correction based on the source packets correctly received at the end of slot $I$ and the corresponding recovery packets correctly received at the end of slot $I+1$. The video packets after correction is then played back. 
TABLE II

START-UP DELAY CONSTRAINT, RECEIVER BANDWIDTH AND $n$ FOR A Client, With PACKet SizE of 1000 Bytes

\begin{tabular}{ccc}
\hline Delay $(\mathrm{s})$ & BW $(\mathrm{kb} / \mathrm{s})$ & $n$ \\
\hline 0.5 & 200 & 6 \\
0.5 & 500 & 15 \\
1.0 & 500 & 31 \\
2.0 & 500 & 62 \\
2.0 & 1000 & 125 \\
10.0 & 500 & 312 \\
\hline
\end{tabular}

5) The time is now advanced to slot $I+1$, and the process repeats.

Clearly, the maximum delay of this scheme is two slots, which is also the start-up delay. We show in Table II the relationship among start-up delay, end-to-end receiver bandwidth and $n$, given a packet size of 1000 bytes and negligible join and leave latencies. We clearly see that $n$ may range from the order of 10 to 100 , depending on the applications. If join and leave latency is nonnegligible, $n$ would be correspondingly reduced.

Note that the receiver may subscribe to more than one copy of its lost packets, i.e., $n_{R} \geq l$. In this case, because the loss is independent and random, to maximize the recovery probability, the number of copies should be the same for all the lost packets. More specifically, let $m_{1}=\left\lfloor n_{R} / l\right\rfloor$ and $m_{2}=\left\lceil n_{R} / l\right\rceil$ be two consecutive integers. Further let $c_{1}$ and $c_{2}$ be the number of lost packets with copies $m_{1}$ and $m_{2}$, respectively. We have

$$
\begin{aligned}
c_{1}+c_{2} & =l \\
m_{1} c_{1}+m_{2} c_{2} & =n_{R} .
\end{aligned}
$$

These equations yield that the receiver gets $m_{2}$ copies of $c_{2}=$ $n_{R}-m_{1} l$ lost packets and $m_{1}$ copies of the remainder of the lost packets.

\section{Problem Formulations}

Given the system operation, we formulate and solve the following two problems.

1) Server Transmission: The server designs its menu for a target receiver experiencing a certain loss probability $\hat{p}(\hat{p}$ may be the maximum loss rate the receivers are likely to experience). Note that $\hat{p}$ can be much higher than $p$, the actual loss rate experienced by any receiver in the system. We assume that the target receiver always uses the optimal combination of FEC and $\mathrm{ReD}$ packets so as to minimize its bandwidth. The objective is to come up with a menu such that the target receiver meet a certain residual error rate $\varepsilon_{0}$. The server transmission problem is hence formulated as

Given : $\hat{p}$

Find : $\left\{N_{F}, M\right\}$

Such that: $\varepsilon \leq \varepsilon_{0}$ for the target receiver.

2) Receiver Selection: Given a server menu $\left(N_{F}, M\right)$, a receiver with bandwidth $n$ and $l$ lost packets has to determine the optimal pair $\left(n_{F}^{*}, n_{R}^{*}\right)$ (both functions of $l$ ) so as to minimize its residual loss rate $\varepsilon$. The problem of receiver selection is hence posed as

$$
\begin{aligned}
\text { Given : } & N_{F}, M, p, n, l \\
\text { Minimize : } & \varepsilon(l) \\
\text { Such that : } & n_{F} \leq \min \left(N_{F}, n-K\right) ; \\
& n_{R} \leq \min (M l, n-K)
\end{aligned}
$$

where the last set of inequalities is due to receiver bandwidth constraint and what is available in the server menu.

\section{ANALYSis ON RESIDUAL ERROR RATE}

In this section, we analyze the overall residual error rate $\varepsilon$ given a loss probability $p$. Clearly, in server transmission problem, the loss probability $p$ is replaced by $\hat{p}$ for the target receiver; for a receiver, the loss probability $p$ is the local measured value for the slot. We show some of the important nomenclature used in this paper in Table III.

Let $\varepsilon(l)$ be the receiver's residual error rate given $l$ losses of the source packets. By conditioning on $l$ and noting that $l$ is binomial-distributed, we have

$$
\varepsilon=\sum_{l=0}^{K}\left(\begin{array}{c}
K \\
l
\end{array}\right)(1-p)^{K-l} p^{l} \cdot \varepsilon(l) .
$$

Let $G_{l}$ be the total number of source packets received in a slot after error correction, given $l$ losses in the source packets. Clearly,

$$
\varepsilon(l)=\frac{K-E\left[G_{l}\right]}{K} .
$$

From (3) and (4), $\varepsilon$ can be obtained once we know $E\left[G_{l}\right]$, which we discuss next.

Let $F$ be the random variable of the number of FEC packets correctly received $\left(0 \leq F \leq n_{F}\right)$. Since each of the packets are lost independently with probability $p$, we have

$$
P(F=f)=\left(\begin{array}{c}
n_{F} \\
f
\end{array}\right)(1-p)^{f} p^{n_{F}-f} .
$$

Further let $Q$ be the number of distinct source packets correctly received due to $\operatorname{ReD}$ packets in the block $(0 \leq Q \leq l)$. Clearly, if $Q+F \geq l$, all losses are repaired; otherwise, the FEC packets received are useless. Therefore, $G_{l}$ is given by

$$
G_{l}= \begin{cases}K, & \text { if } Q+F \geq l \\ K-l+Q, & \text { otherwise }\end{cases}
$$

Therefore,

$$
\begin{aligned}
E\left[G_{l}\right]= & K \cdot P(Q+F \geq l) \\
& +(K-l+E[Q \mid Q+F<l]) \times P(Q+F<l) \\
= & K+(E[Q \mid Q+F<l]-l) \times P(Q+F<l) .(7)
\end{aligned}
$$

In order to obtain $E[Q \mid Q+F<l]$ and $P(Q+F<l)$, we need to consider two cases: $n_{R}<l$ (single copy of a lost packet) and $n_{R} \geq l$ (multiple copies of a lost packet). 
TABLE III

NOMENCLATURE USED IN THIS PAPER

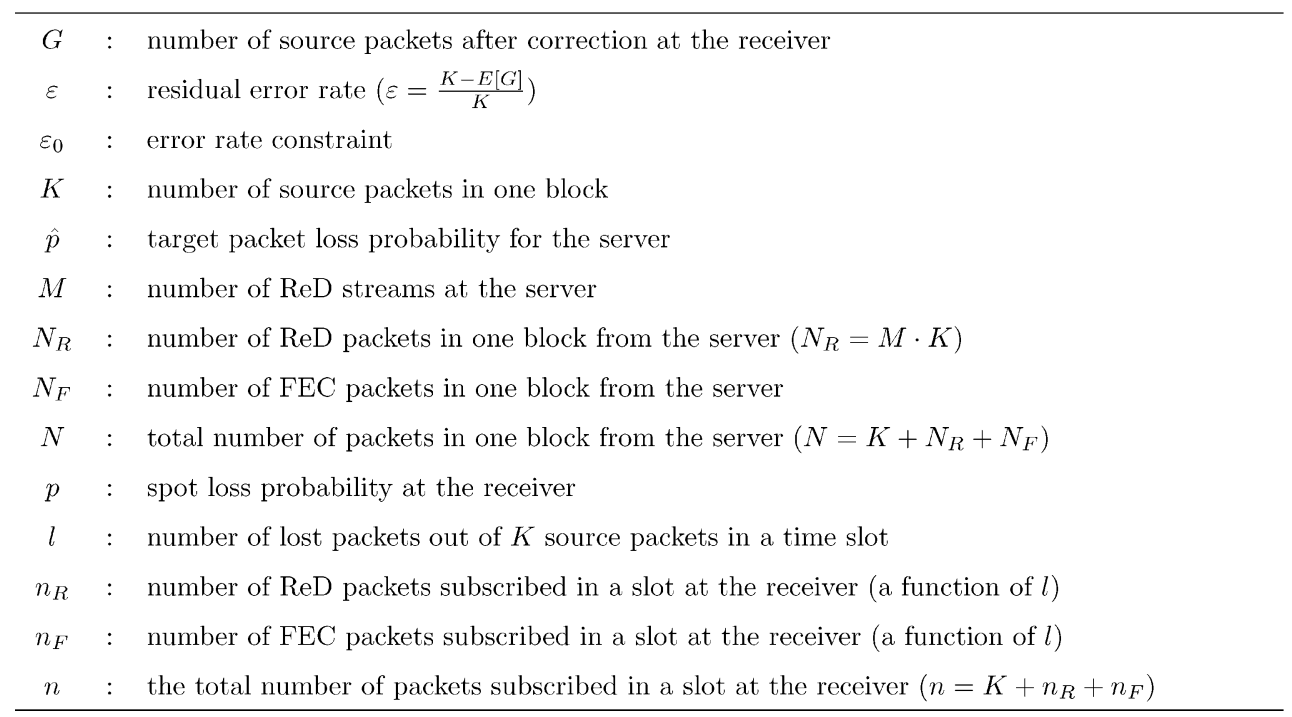

- Case I: $n_{R}<l$

In this case, the receiver subscribes a single copy of $n_{R}$ lost source packets. Since all the ReD packets are distinct, we have

$$
P(Q=q)=\left(\begin{array}{c}
n_{R} \\
q
\end{array}\right)(1-p)^{q} p^{n_{R}-q} .
$$

Therefore,

$$
\begin{aligned}
E[Q \mid Q+F<l] & =\sum_{q=0}^{n_{R}} q \cdot P(Q=q \mid Q+F<l) \\
& =\frac{\sum_{q=0}^{n_{R}} q P(Q=q) P(F<l-q)}{P(Q+F<l)}
\end{aligned}
$$

where

$$
\begin{aligned}
P(Q+F<l)= & \sum_{q=0}^{n_{R}} P(Q=q) \cdot P(F<l-q) \\
= & \sum_{q=0}^{n_{R}}\left\{\begin{array}{l}
n_{R} \\
q \\
q=0
\end{array}\right. \\
& \times\left[\sum_{f=0}^{l-q-1}\left(\begin{array}{c}
n_{F} \\
f
\end{array}\right)(1-p)^{n_{R}-q} p^{n_{F}-f}\right] .
\end{aligned}
$$

- $\quad$ Case II: $n_{R} \geq l$

The receiver subscribes multiple copies of the lost source packets. Let the number of copies be $m$, where $m=$ $n_{R} / l$. For simplicity, we consider $m$ a real number. (We have done an exact analysis on using integral $m$, i.e., $m_{1}$ and $m_{2}$ as in (1) and (2) and the results are very similar. See Appendix A for the detailed analysis.) To recover a lost packet, at least one out of the $m$ copies has to be received correctly, which is with probability $\alpha \equiv 1-p^{m}$. Therefore

$$
\begin{aligned}
P(Q=q) & =\left(\begin{array}{l}
l \\
q
\end{array}\right) \alpha^{q}(1-\alpha)^{l-q} \\
& =\left(\begin{array}{l}
l \\
q
\end{array}\right)\left(1-p^{m}\right)^{q} p^{m(l-q)} .
\end{aligned}
$$

Furthermore, we have

$$
\begin{aligned}
E[Q \mid Q+F<l] & =\sum_{q=0}^{l-1} q \cdot P(Q=q \mid Q+F<l) \\
& =\frac{\sum_{q=0}^{l-1} q P(Q=q) P(F<l-q)}{P(Q+F<l)}
\end{aligned}
$$

where

$$
P(Q+F<l)=\sum_{q=0}^{l-1} P(Q=q) \cdot P(F<l-q),
$$

and

$P(F<l-q)=\left[\sum_{f=0}^{l-q-1}\left(\begin{array}{c}n_{F} \\ f\end{array}\right)(1-p)^{f} p^{n_{F}-f}\right]$.

Equation (9) or (11) can then be used to obtain $E\left[G_{l}\right]$ in (7), which in turn can be used to obtain $\varepsilon(l)$ in (4). $\varepsilon(l)$ then finally leads to $\varepsilon$ as given in (3).

Given these expressions, we can then solve server transmission and client selection problems as follows.

- Server transmission This problem can be solved with Algorithm 1 based on exhaustive search. By noting that $\varepsilon$ decreases with increasing $n$, we increase $n$ from $K$ until $\varepsilon \leq \varepsilon_{0}$ is reached. This is what the first outer loop does in the algorithm. For a given $n$ and $l$, we search for the optimal $n_{R}^{*}(l)$, and hence $n_{F}^{*}(l)=n-K-n_{R}^{*}(l)$, to achieve the minimum $\varepsilon(l)$ (the innermost for loops). The 
computed $\left(n_{R}^{*}(l), n_{F}^{*}(l)\right)$ pair is then used to calculate the server menu $\left(N_{F}, M\right)$, where $N_{F}$ and $N_{R}$ are given by

$$
\begin{aligned}
N_{F} & =\max _{l} n_{F}^{*}(l) \\
M & =\max _{l}\left\lceil\frac{n_{R}^{*}(l)}{l}\right\rceil
\end{aligned}
$$

respectively.

The corresponding server bandwidth is therefore

$$
N=K+N_{F}+M K \text {. }
$$

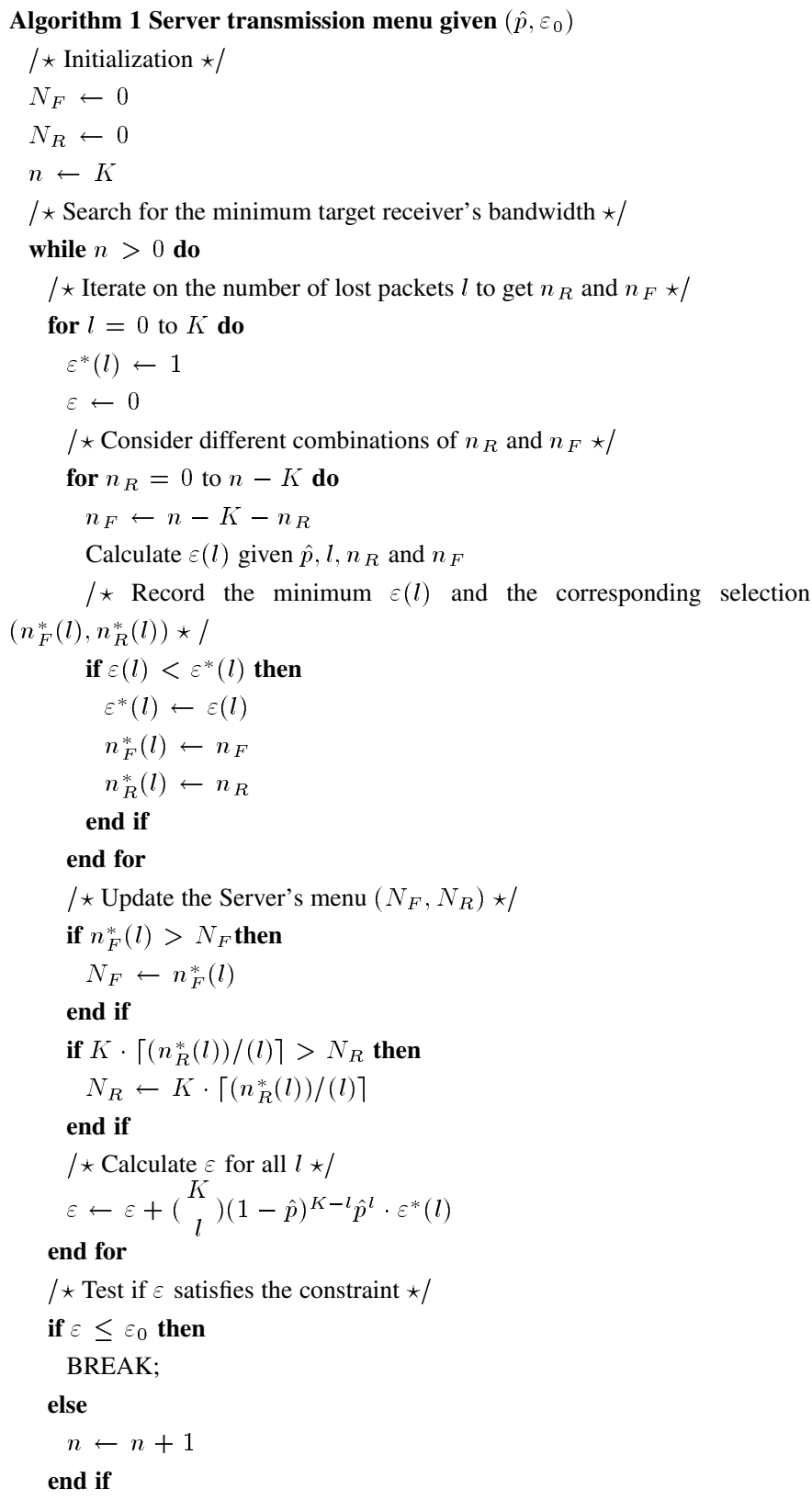

\section{- Receiver selection}

The selection problem can be solved according to Algorithm 2 based on exhaustive search. Given $n$ and $l$, the receiver iterates on $n_{R}$, and hence $n_{F}=n-K-n_{R}$, so as to find a pair $\left(n_{F}^{*}(l), n_{R}^{*}(l)\right)$ which minimizes $\varepsilon(l)$ (the for loop).

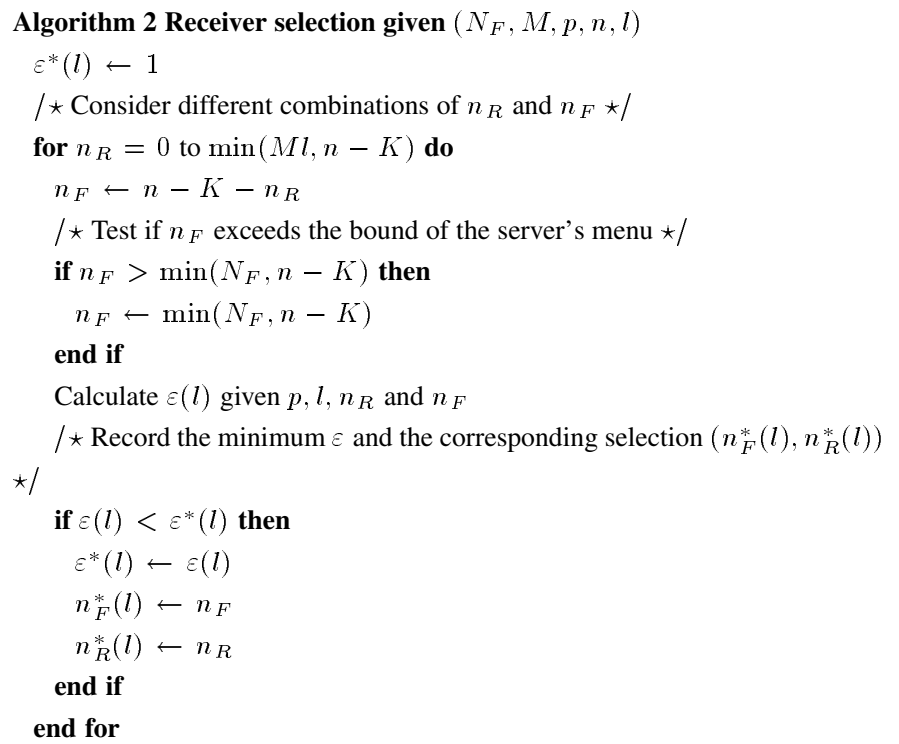

We remark before leaving this section that our equations are also applicable to pure FEC and pure ReD cases by substituting $n_{R}=0$ and $n_{F}=0$, respectively. Doing so, we have, for pure FEC scheme (i.e., $E[Q \mid Q+F<l]=0$ ),

$$
\begin{aligned}
E\left[G_{l}\right] & =K-l \cdot P(F<l) \\
& =K-l \times \sum_{f=0}^{l-1}\left(\begin{array}{c}
n_{F} \\
f
\end{array}\right)(1-p)^{f} p^{n_{F}-f} .
\end{aligned}
$$

For the pure ReD case, we have

$$
G_{l}=K-l+Q
$$

and hence

$$
E\left[G_{l}\right]= \begin{cases}K-l+n_{R}(1-p), & \text { if } n_{R}<l \\ K-l \cdot p^{m}, & \text { otherwise }\end{cases}
$$

\section{FAST AllocAtion Algorithms fOR MULTIPLE VIDEO LAYERS}

Our previous algorithm on receiver selection is based on exhaustive search. Though accurate, it is computationally intensive (it is not hard to see that its time complexity is $O\left(n^{4}\right)$ ). ${ }^{2}$ Since the receiver needs to compute the number of FEC and ReD packets at the slot boundaries, some fast but accurate approach is desirable. This is especially true for receivers with low processing capability such as handhelds. Such fast approximation would also be useful when we have a client of a certain $a g$ gregate bandwidth which needs to be partitioned among video layers efficiently to meet some residual error requirement.

\footnotetext{
${ }^{2}$ The proof is as follows. The algorithm is based on linear search on $n_{R}$, which is of of range $n-K$. Given $l$ lost packets out of $K$ packets, we calculate the receiver's residual error rate $\varepsilon$ for each pair of $\left(n_{R}, n_{F}\right)$. This iteration takes $O(n)$ time. The time to compute combinatorials is $O\left(n^{2}\right)$ (based on triangular computation). Since we are summing up the combinatorials $O(n)$ times [(9) or (11)], the complexity of computing $\varepsilon$ is hence $O\left(n^{3}\right)$. The total complexity for receiver selection is therefore $O\left(n^{4}\right)$. For server menu creation, since it in addition has to run an outer iteration loop to search for an optimal $n$, it is of complexity $O\left(n^{5}\right)$.
} 
In this section, we consider the joint allocation of layer bandwidth, FEC, and ReD packets, such that a certain error requirement in each layer is met. Specifically, we consider a multicasting $L$ video layers, with the base layer labeled as layer 0 , and the enhancement layers labeled as layer $1,2, \ldots, L-1$. Each layer is transmitted independently and in parallel along with its error recovery packets from the server. Each layer may have different loss rate and residual loss requirement, i.e., $\varepsilon_{0}{ }^{(i)}$ for layer $i(0 \leq i \leq L-1)$. In general, $\varepsilon_{0}{ }^{(0)} \leq \varepsilon_{0}^{(1)} \leq \ldots \leq \varepsilon_{0}^{(L-1)}$.

Consider an arbitrary receiver of aggregate bandwidth $n$. Let $n_{i}^{*}$ be the minimum bandwidth of layer $i$ meeting the error requirement of the layer. Clearly, if $S$ is the number of layers the client joins, we have

$$
n \leq \sum_{i=0}^{S-1} n_{i}^{*}
$$

We propose fast algorithms for the following problems.

1) How to determine $n_{i}^{*}$ ? (This problem is analogous to finding the target receiver's minimum bandwidth in the server's transmission problem); and

2) Given $n_{i}^{*}$ and a certain number of losses $l$, how to determine the optimal combination of FEC and ReD packets, $\left(n_{F}^{*}(l), n_{R}^{*}(l)\right)$ ? (This problem is the same as solving the receiver selection problem for a single layer).

Our algorithms are based on closed form expressions, and hence reduces the complexity substantially to $O(1)$. We elaborate more on the algorithms as follows.

\section{A. Fast Approximation of Layer Bandwidth}

In this section, we present how to find an approximated value $n_{i}^{\prime}$ for $n_{i}^{*}$. With loss of generality, consider an arbitrary layer $i$ with bandwidth $K$ and loss probability $p$. We find $n_{i}^{\prime}$ corresponding to the pure FEC scheme (i.e., $n_{F, i}=n_{i}^{\prime}-K$ ). This is clearly a pessimistic case, as our hybrid scheme performs better than pure FEC. We show that the $n_{i}^{\prime}$ derived this way only slightly over-estimates as compared with the approach based on exhaustive search.

Recall from (17) that $E\left[G_{l}\right]=K-l \cdot P(F<l)$. Therefore, from (4),

$$
\begin{aligned}
\varepsilon(l) & =\frac{K-E\left[G_{l}\right]}{K} \\
& \approx p(1-P(F \geq l))
\end{aligned}
$$

where we have used, for simplicity, a deterministic model that $l=K \cdot p$. Assuming that $\varepsilon(l) \approx \varepsilon_{0}{ }^{(i)}$, we have

$$
P(F \geq l) \approx \frac{p-\varepsilon_{0}{ }^{(i)}}{p} .
$$

Note that $F$ is a random variable of binomial distribution, which we further approximate by a Gaussian distribution with mean $n_{F, i}(1-p)$ and standard deviation $n_{F, i} p(1-p)$ (this approximation is good when $n_{F, i}$ is reasonably large, say greater than 5). Therefore,

$$
P(F \geq l) \approx P\left(Z \geq \frac{l-n_{F, i}(1-p)}{\sqrt{n_{F, i}(1-p) p}}\right)
$$

where $Z$ is a random variable of a normal distribution with mean 0 and standard deviation 1 .

It is well-known that the tail of the normal distribution $P(Z>$ $z$ ) can be approximated by an exponential function [44], i.e.,

$$
f_{1}(z) \leq P(Z>z) \leq f_{2}(z)
$$

where

and

$$
f_{1}(z)=\frac{\sqrt{\pi / 2}}{\sqrt{z^{2}+2 \pi}+(\pi-1) z} e^{-z^{2} / 2},
$$

$$
f_{2}(z)=\frac{\sqrt{\pi / 2}}{\sqrt{(\pi-2)^{2} z^{2}+2 \pi}+2 z} e^{-z^{2} / 2} .
$$

We may equivalently write

$$
P(Z \geq z)=c_{0}(z) e^{-z^{2} / 2}
$$

for some function $c_{0}(z)$. Through experiments, we find that $c_{0}(z) \approx c_{0}=3$ (a constant) for most of the cases of our interest. Therefore, using (22), we have

$$
\begin{aligned}
P\left(Z \geq \frac{l-n_{F, i}(1-\hat{p})}{\sqrt{n_{F, i}(1-\hat{p}) \hat{p}}}\right) & =c_{0} \exp \left(-\frac{\left[l-n_{F, i}(1-\hat{p})\right]^{2}}{2 n_{F, i}(1-\hat{p}) \hat{p}}\right) \\
& \approx \frac{\hat{p}-\varepsilon_{0}{ }^{(i)}}{\hat{p}}
\end{aligned}
$$

which can be solved for $n_{F, i}$.

Setting $l=K p$, we obtain the larger root of the above equation as the approximated value for $n_{F, i}$ and set $n_{i}^{\prime}=K+n_{F, i}$. The number of layers $S$ the receiver joins is such that

$$
\sum_{i=0}^{S} n_{i}^{\prime} \leq n<\sum_{i=0}^{S+1} n_{i}^{\prime}
$$

\section{B. Fast Approximation on the Optimal Selection Given $N_{i}$}

In this section, we present a fast approximation for the optimal selection of FEC and ReD packets given layer bandwidth $n_{i}, l$, and the loss probability of the layer $p$.

To derive the approximation $n_{R, l}^{\prime}$ for $n_{R}^{*}(l)$, we first observe from (6) that $G_{l}=K-l+Q \approx K-l+n_{R, l}^{\prime}(1-p)$ (based on the deterministic model on $Q$ ). Since the residual error rate is low, we have $G_{l} \approx K$, which yields $n_{R, l}^{\prime}=(l) /(1-p)$. Taking into the account the constraint on the maximum number of recovery packets (given by $n-K$ ) and the server menu constraint (given by $M l$ ), we therefore have

$$
n_{R, l}^{\prime}=\min \left\{\left\lfloor\frac{l}{1-p}\right\rfloor, n-K, M l\right\} .
$$

The corresponding approximation $n_{F, l}^{\prime}$ for $n_{F}^{*}(l)$ is hence

$$
n_{F, l}^{\prime}=\min \left\{n-K-n_{R}^{\prime}, N_{F}\right\} .
$$

Note that (26) and (27) are based on closed form expressions without any iterative search and hence are of complexity $O(1)$.

\section{ILLUSTRATIVE NUMERICAL RESULTS}

In this section, we present illustrative numerical results for server transmission and receiver selection problems, using the baseline parameters $\varepsilon_{0}=4 \%, K=30$ (corresponding to a 


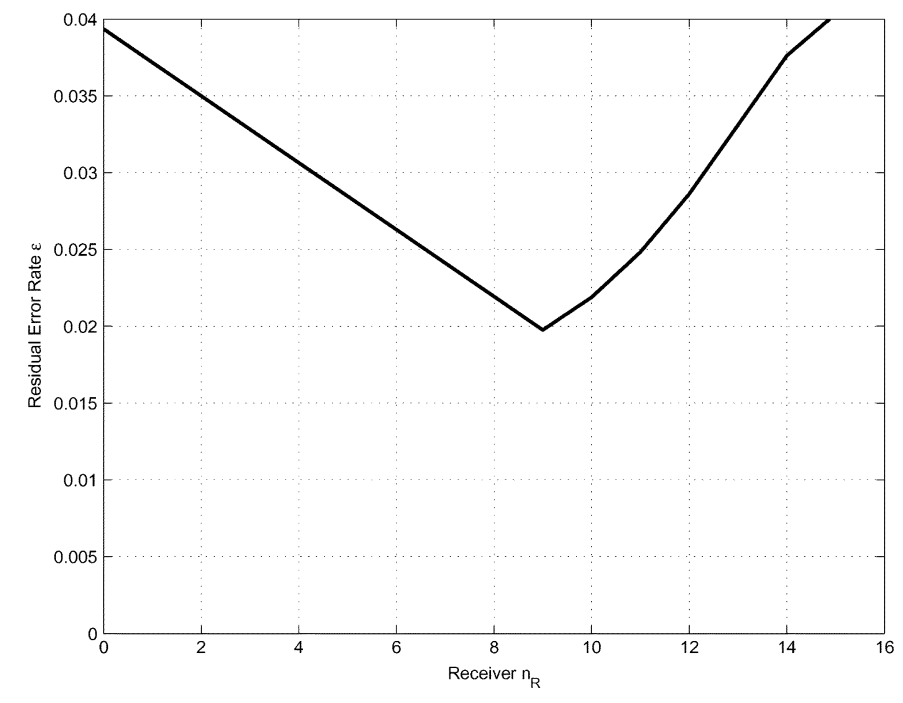

Fig. 3. $\varepsilon$ versus $n_{R}$ for a target receiver $(K=30, \hat{p}=0.4, n=45$, and $l=9)$.

startup delay of one to two seconds in Table II), and $\hat{p}=0.4$ (which means that we design the server menu with the maximum loss rate among all the receivers at any time being 0.4). Note that the actual receiver loss rate $p$ may be much lower than $\hat{p}$. We vary some of these parameters while keeping the rest the same in our plots. We first present results for the exact analysis, and finally we show how well our fast algorithms agree with the exact analysis. In Sections V-A and V-B, we consider single layer video, while in Section V.C we consider multiple video layers.

\section{A. Server Transmission}

We first show that there is indeed an optimal mix to minimize $\varepsilon$. We show in Fig. $3 \varepsilon$ versus $n_{R}$ for a receiver with $l=9$ lost packets. Recall that $n_{F}=n-K-n_{R}$; therefore, as $n_{R}$ increases, $n_{F}$ correspondingly decreases. As $n_{R}$ increases, $\varepsilon$ first decreases to a minimum and then increases, showing clearly the trade-off between FEC and ReD packets. There is indeed an optimal mix $\left(n_{R}^{*}, n_{F}^{*}\right)$ to achieve the minimum $\varepsilon$. The plot shows that the optimal policy would be first to recover partially the lost packets with the use of ReD packets, and then use FEC to repair the rest. Note that the case $n_{R}=0$ corresponds to the pure FEC case while the case $n_{R}=n-K=15$ corresponds to the pure ReD case. A mix of RED and FEC packets can achieve a substantial reduction in $\varepsilon$ as compared to the pure policies (cut by $50 \%$ here).

Fig. 4 shows the composition of $\left(N_{F}, N_{R}=\mathrm{MK}\right)$ in server transmission against $\hat{p}$. When $\hat{p}$ increases, both $N_{R}$ and $N_{F}$ increase in order to reduce the residual loss to the target $\varepsilon_{0}$. The discrete jumps in the recovery packets is due to a new replicated stream, i.e., a jump of $K$ packets $\left(N_{R}\right.$ is an integer multiple of $K$ ). The more gentle increase in bandwidth after each jump is due to the increase in FEC. For our baseline of $\hat{p}=0.4$, the server provides $M=2$ replicated delayed streams and $N_{F}=25$ FEC packets. The figure clearly shows replicating streams once or twice can effectively reduce the residual loss rate of the receivers.

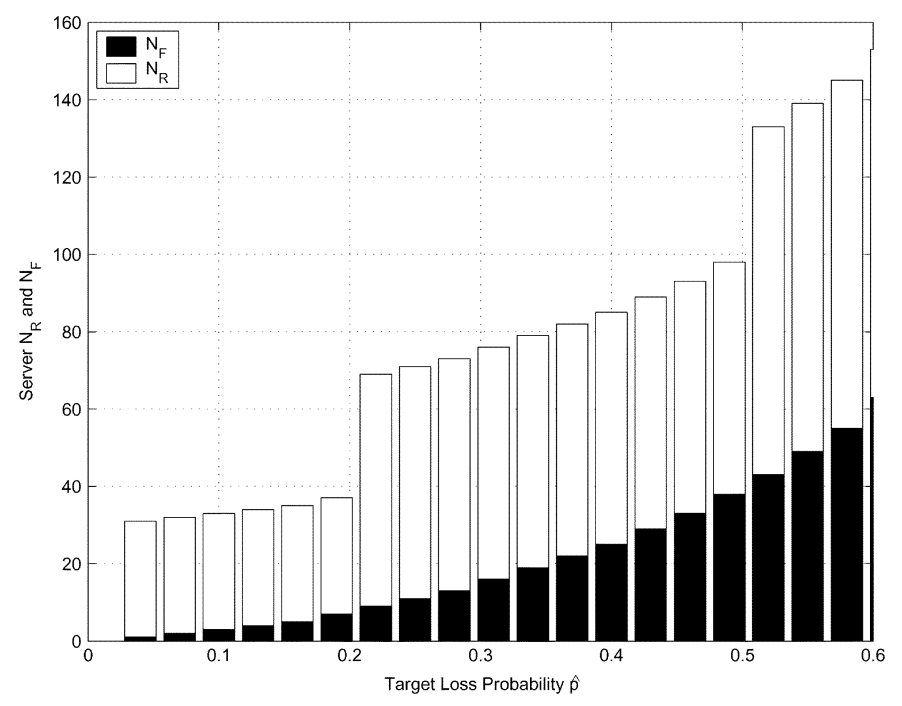

Fig. 4. Server's menu $N_{R}=\mathrm{MK}$ and $N_{F}$ versus $\hat{p}\left(K=30\right.$, and $\left.\varepsilon_{0}=4 \%\right)$.

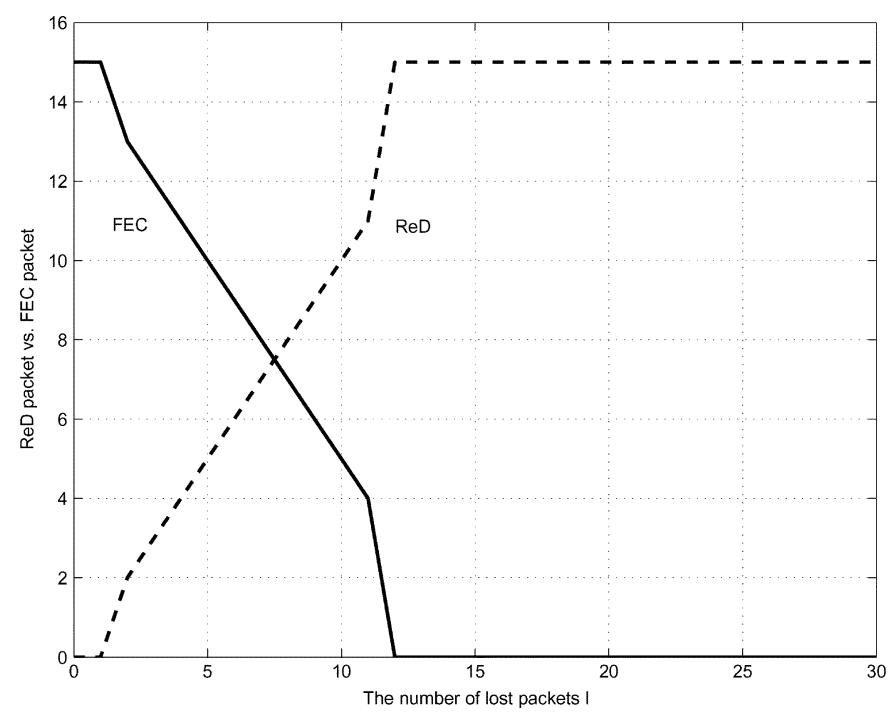

Fig. 5. Optimal combination of FEC and ReD packets versus $l$ for a receiver $\left(K=30, \varepsilon_{0}=4 \%, M=2, N_{F}=25, n=45\right.$, and $\left.p=0.2\right)$.

\section{B. Receiver Selection}

In this section, we present results for receiver selection problem. In Fig. 5, we plot the optimal selection $n_{R}^{*}(l)$ and $n_{F}^{*}(l)$ versus the number of losses $l$ for a receiver with $p=0.2$ and $n=45$ when the server is designed with $\hat{p}=0.4$ (i.e., with $M=2, N_{F}=25$ ). Clearly, when $l$ is low, the optimal policy would be just to subscribe FEC packets. On the other hand, when $l$ is high, the optimal policy would be pure ReD packets. For intermediate $l$, the optimal policy would be a mix of FEC and RED packets.

We next compare in Fig. 6 the resultant $\varepsilon$ for pure FEC, pure $\mathrm{ReD}$, and our hybrid scheme versus $p(n=45)$. Clearly, $\varepsilon$ increases with $p$. There is a point of $p$ beyond which $\varepsilon$ increases rapidly. We see that when $p$ is low, pure FEC is more effective than pure ReD. On the other hand, when $p$ is high, pure $\operatorname{ReD}$ scheme outperforms pure FEC scheme. Our scheme traces the lower envelop of both curves and performs the best. It substantially reduces the residual error rate, especially for intermediate 


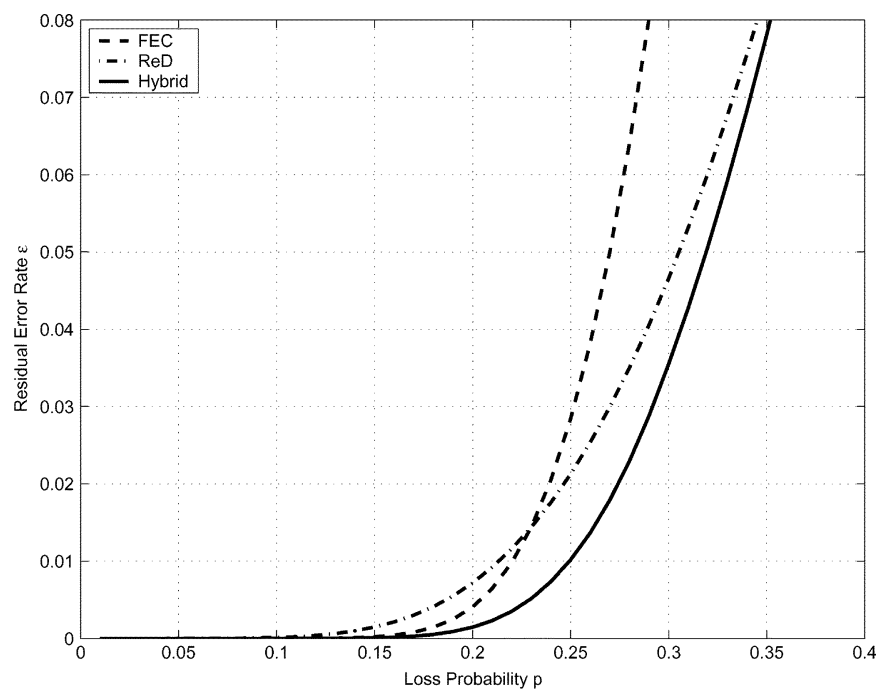

Fig. 6. Residual error rate of a client for pure FEC, pure ReD and our hybrid scheme.

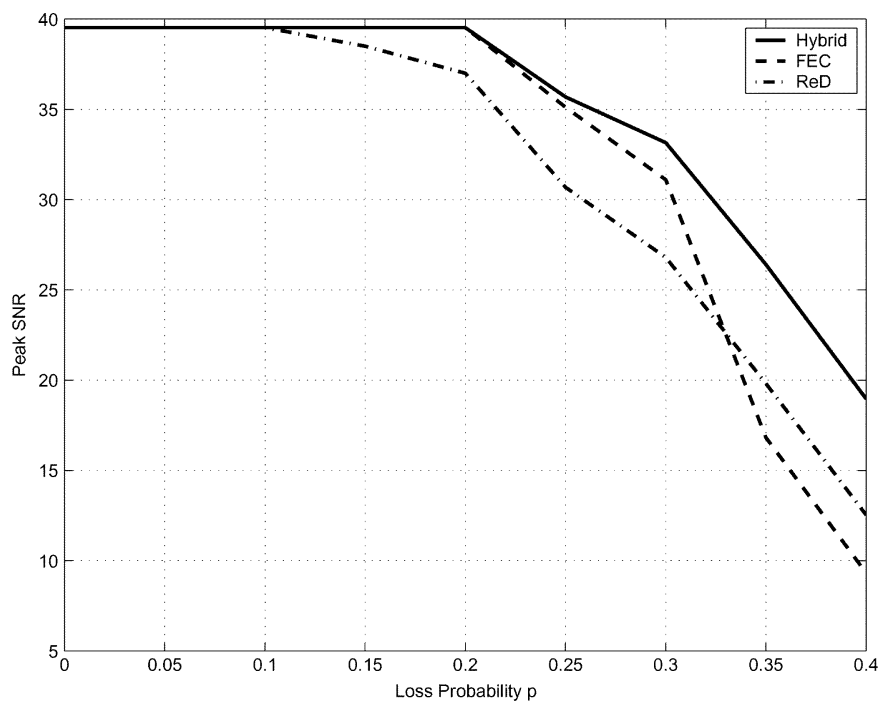

Fig. 7. Objective comparison between pure FEC, pure ReD, and our hybrid scheme $\left(K=30, \varepsilon_{0}=4 \%, \hat{p}=0.4, n=45, \mathrm{GoP}=8, Q P=3\right.$, and a packet size of 1024 bytes).

$p$. For example, when $p=0.25$, our scheme reduces $\varepsilon$ by almost half.

We finally compare the three schemes in terms of the resultant video quality by using H.263+ video sequences. In Fig. 7 , we present the objective comparison among the schemes with the foreman sequence, where the PSNR values of the decoded video sequence versus $p$ is plotted $(\mathrm{GoP}=8, \mathrm{QP}=3$ and a packet size of 1024 bytes). When $p$ is small, the three schemes have similar PSNR due to low residual error. As $p$ increases, the PSNR, and hence video quality, degrades. The pure FEC scheme performs better than the pure $\operatorname{ReD}$ scheme when $p$ is low, while the ReD scheme out-performs FEC when $p$ is high. Once again, the PSNR of the hybrid scheme is higher than the other two schemes (by many $\mathrm{dB}$ ). It is especially significant as $p$ increases.

We show the subjective comparison of a typical video frame from the pure FEC and hybrid schemes in Fig. 8(a) and (b), respectively $(p=0.25)$. Clearly, the visual quality of the video using pure FEC scheme is much worse than the hybrid scheme.

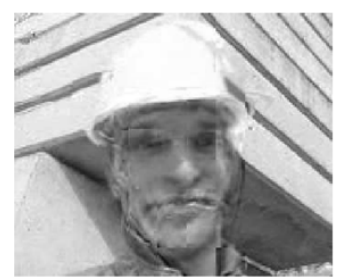

a)

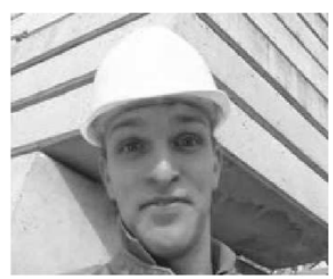

b)
Fig. 8. Subjective comparison of foreman video sequence using pure and hybrid schemes $\left(p=0.25, K=30, \varepsilon_{0}=4 \%, \hat{p}=0.4, n=45, \mathrm{GoP}=\right.$ $8, Q P=3$, and a packet size of 1024 bytes). (a) Pure FEC schem. (b) Hybrid scheme.

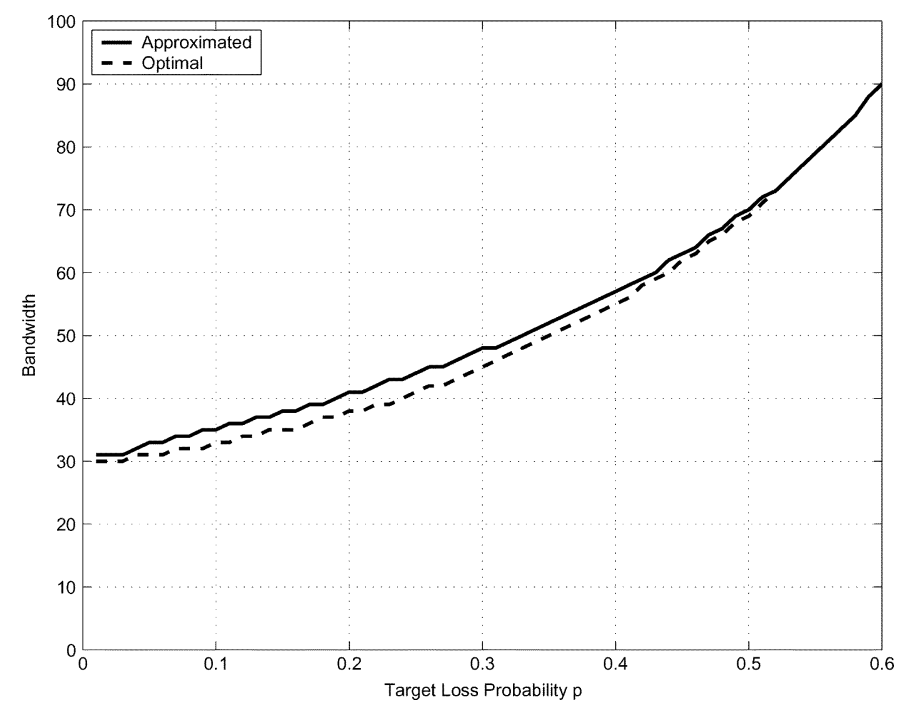

Fig. 9. Comparison between $n_{i}^{*}$ and $n_{i}^{\prime}$ versus $p\left(K=30, \varepsilon_{0}{ }^{(i)}=4 \%\right.$, and $\left.c_{0}=3\right)$.

\section{Fast Allocation Algorithms}

In this section, we show how close our approximation algorithms are as compared to the exact analysis. We plot $n_{i}^{\prime}$ and $n_{i}^{*}$ versus $p$ in Fig. 9 based on the solving of (24). The two curves are very close, indicating that our approximation is very good. Our approximation slightly over-estimates the optimal value due to the fact that it is based on the more pessimistic pure FEC scheme.

Using the approximated pair $\left(n_{F}^{\prime}, n_{R}^{\prime}\right)$ as given in (26) and (27), we can calculate the corresponding residual error rate for the receiver. We compare the resultant residual error rate using our approximation and the optimal $\varepsilon^{*}$ based on exhaustive search in Fig. 10, given a server menu designed with $\hat{p}=0.4$ ( $n=45, M=2$, and $N_{F}=25$ ). Clearly, our approximation is very close, indicating that it is good.

\section{CONCLUSION}

In video multicast, packet losses have to be mitigated in order to offer good-quality video. In this paper, we propose and study a feedback-free loss recovery scheme which combines FEC with stream replication. The server multicasts FEC and replicated delayed (ReD) streams to clients, and the clients, depending on their local loss rate, autonomously and dynamically subscribe to these recovery packets to repair their losses. We consider that all packets are subject to losses. For the server, we study how many replicated streams and FEC packets are necessary to meet 


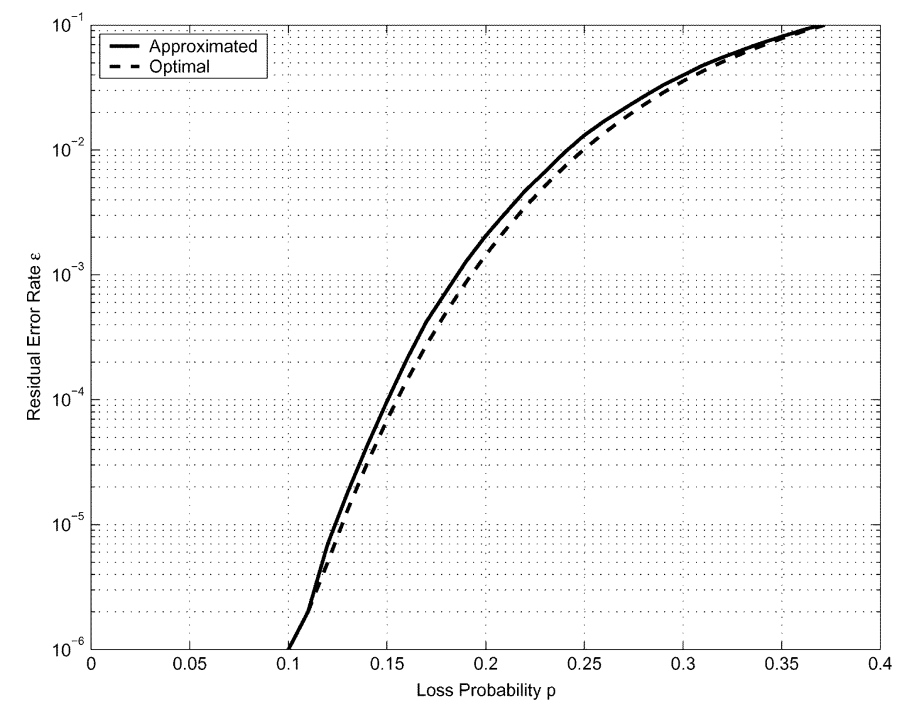

Fig. 10. Comparison of residual error rates versus $p$ for receiver selection based on exhaustive search (optimal) and approximation $\left(K=30, \varepsilon_{0}=\right.$ $4 \%, M=2, N_{F}=25$, and $\left.n=45\right)$.

a certain residual loss requirement, while for the receiver, we investigate the optimal mix of FEC and ReD packets to minimize residual loss. We finally present fast but accurate algorithms for the receivers on the joint allocation of layer bandwidths, FEC and $\mathrm{ReD}$ packets so as to meet a certain error requirement for each layer.

We have presented an analysis of the proposed system. Our results show that, given a certain server bandwidth, the residual loss rate can be effectively reduced if both FEC and ReD streams are used. The optimal recovery policy is a mixture of FEC and ReD packets: the ReD packets are used to recover partially the set of lost packets, and FEC is used to repair the rest. This policy achieves a substantial reduction in the residual error rate as compared to pure FEC or pure ReD alone (by more than $50 \%$ in our experiments). Our fast approximation algorithms for joint allocation are based on direct closed-form expressions and hence are of much lower complexity (reduced from $O\left(n^{5}\right)$ to $O(1)$ for allocation of layer bandwidth, and from $O\left(n^{4}\right)$ to $O(1)$ for optimal FEC and ReD packets allocation, where $n$ is the number of packets received in the startup delay of the system). Our approximation algorithms achieve similar performance as the exact solutions based on direct computation and exhaustive search.

\section{APPENDIX I \\ EXACT ANALYSIS ON $n_{R} \geq l$}

Here we present the exact analysis of the system for the case $n_{R} \geq l$. Recall that the receiver subscribes $m_{1}$ copies of $\mathrm{ReD}$ packets for $c_{1}$ lost packets and $m_{2}$ copies for $c_{2}$ lost packets, where $m_{1}=\left\lfloor n_{R} / l\right\rfloor, m_{2}=\left\lceil n_{R} / l\right\rceil, c_{1}=l-\left(n_{R}-m_{1} l\right)$, and $c_{2}=n_{R}-m_{1} l$. Let $Q_{1}$ and $Q_{2}$ be the random variables of received packets out of the $c_{1}$ and $c_{2} \operatorname{ReD}$ packets, respectively. Clearly,

$$
\begin{aligned}
& P\left(Q_{1}=q_{1}\right)=\left(\begin{array}{l}
c_{1} \\
q_{1}
\end{array}\right)\left(1-p^{m_{1}}\right)^{q_{1}}\left(p^{m_{1}}\right)^{c_{1}-q_{1}} \\
& P\left(Q_{2}=q_{2}\right)=\left(\begin{array}{l}
c_{2} \\
q_{2}
\end{array}\right)\left(1-p^{m_{2}}\right)^{q_{2}}\left(p^{m_{2}}\right)^{c_{2}-q_{2}} .
\end{aligned}
$$

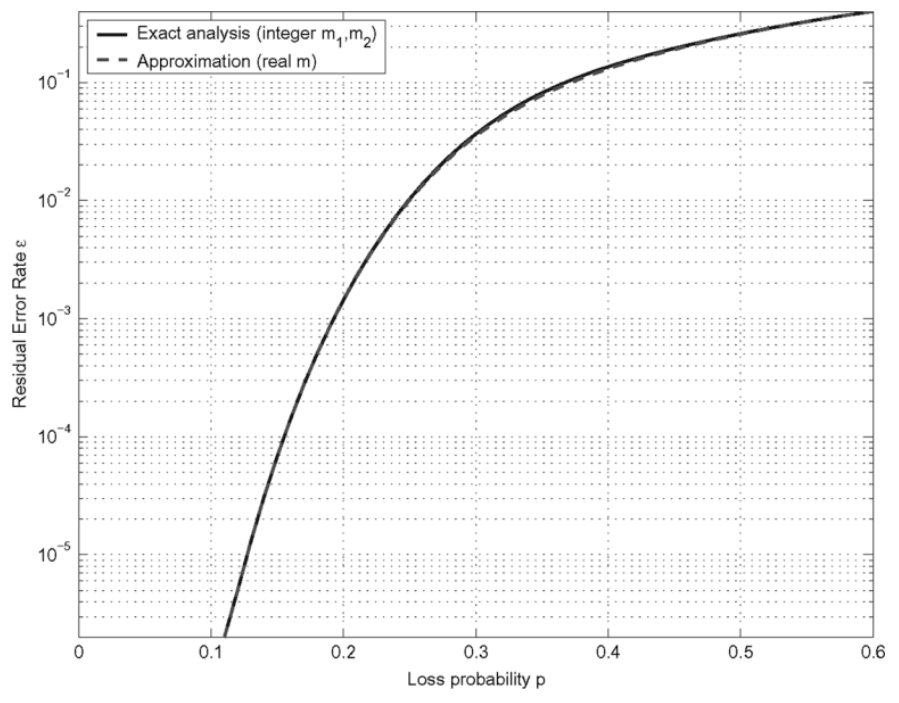

Fig. 11. Residual error rates vs. receiver loss rate $p$ with integral or real $m$ $\left(K=30, \varepsilon_{0}=4 \%, M=2, N_{F}=25\right.$, and $\left.n=45\right)$.

Therefore,

$$
P(Q=q)=\sum_{q_{1}=0}^{c_{1}} P\left(Q_{1}=q_{1}\right) P\left(Q_{2}=q-q_{1}\right) .
$$

Equations (11)-(13) remain the same as before. In Fig. 11, we show the comparison of residual error rate between the cases using the $m$ based on real number (Section III) and based on using integral $m$ (i.e., using $m_{1}$ and $m_{2}$ above) for a receiver with loss rate $p$. Clearly, there is little difference between them. Therefore, $m$ may be taken as continuous for simplicity, as in our previous analysis.

\section{REFERENCES}

[1] S. Deering, "Internet multicast routing: State of the art and open research issues," in Multimedia Integrated Conferencing for Europe (MICE) Seminar. Stockholm, Oct. 1993.

[2] G. Carle and E. Biersack, "Survey of error recovery techniques for IP-based audio-visual multicast applications," IEEE Network, vol. 11, pp. 24-36, Nov.-Dec. 1997.

[3] D. Wu, Y. T. Hou, W. Zhu, Y.-Q. Zhang, and J. Peha, "Streaming video over the internet: Approaches and directions," IEEE Trans. Circuits Syst. Video Technol., vol. 11, no. 3, pp. 282-300, Mar. 2001.

[4] Y. Wang and Q.-F. Zhu, "Error control and concealment for video communication: A review," Proc. IEEE, vol. 86, no. 5, pp. 974-997, May 1998.

[5] B. Girod and N. Farber, "Feedback-based error control for mobile video transmission," Proc. IEEE, vol. 87, pp. 1707-23, Oct. 1999.

[6] Y. Ishibashi, Y. Tachibana, and S. Tasaka, "Responsiveness of layered multicast and feedback control for video traffic in the Internet," in Proc. IEEE ICC, vol. 2, 2000, pp. 846-52.

[7] W. Li, "Overview of fine granularity scalability in MPEG-4 video standard," IEEE Trans. Circuits Syst. Video Technol., vol. 11, pp. 301-317, Mar. 2001.

[8] F. Wu, S. Li, and Y.-Q. Zhang, "A framework for efficient progressive fine granulairity scalable video coding," IEEE Trans. Circuits Syst. Video Technol., vol. 11, pp. 332-344, Mar. 2001.

[9] S. Floyd, V. Jacobson, C. Liu, S. McCanne, and L. Zhang, "A reliable multicast framework for light-weight sessions and application level framing," IEEE Trans. Networking, vol. 5, pp. 784-803, Dec. 1997.

[10] S. Paul, K. Sabnani, J. Lin, and S. Bhattacharyya, "Reliable multicast transport protocol (RMTP)," IEEE J. Sel. Areas Commun., vol. 15, pp. 407-421, Apr. 1997.

[11] J. Nonnenmacher, L. Martin, J. Matthias, E. Biersack, and G. Carle, "How bad is reliable multicast without local recovery," in Proc. IEEE INFOCOM, vol. 3, Mar. 1998, pp. 972-979. 
[12] I. Rhee, S. R. Joshi, M. Lee, S. Muthukrishnan, and V. Ozdemir, "Layered multicast recovery," in Proc. IEEE INFOCOM, vol. 2, 2000, pp. 805-813.

[13] S. Kasera, J. F. Kurose, and D. Towsley, "A comparison of server-based and receiver-based local recovery approaches," in Proc. IEEE INFOCOM, Mar. 1998, pp. 988-995.

[14] L. Vicisano, J. Crowcroft, and L. Rizzo, "TCP-like congestion control for layered multicast data transfer," in Proc. IEEE INFOCOM, vol. 3, 1998, pp. 996-1003.

[15] J. Byers, M. Luby, M. Mitzenmacher, and A. Rege, "A digital fountain approach to reliable distribution of bulk data," in Proc. ACM SIGCOMM, 1998, pp. 56-67.

[16] X. Li, M. Ammar, and S. Paul, "Video multicast over the Internet," IEEE Network, vol. 13, pp. 46-60, Mar.-Apr. 1999.

[17] C. Papadopoulos, G. Parulkar, and G. Varghese, "An error control scheme for large-scale multicast applications," in Proc. IEEE INFOCOM, vol. 3, 1998, pp. 1188-1196.

[18] P. Chou, A. Mohr, A. Wang, and S. Mehrotra, "Error control for receiverdriven layered multicast of audio and video," IEEE Trans. Multimedia, vol. 3, no. 1, pp. 108-122, Mar. 2001.

[19] W.-T. Tan and A. Zakhor, "Video multicast using layered FEC and scalable compression," IEEE Trans. Circuits Syst. Video Technol., vol. 11, no. 3, pp. 373-86, Mar. 2001.

[20] J. Cai, Q. Zhang, W. Zhu, and C. Chen, "An FEC-based error control scheme for wireless MPEG-4 video transmission," in Proc. Wireless Communications and Networking Conf., vol. 3, 2000, pp. 1243-1247.

[21] T.-W. A. Lee, S.-H. G. Chan, Q. Zhang, W. W. Zhu, and Y. Q. Zhang, "Optimal allocation of packet-level and byte-level FEC in video multicasting over wired and wireless networks," in Proc. IEEE Globecom, vol. 3, Nov. 2001, pp. 1994-1998.

[22] _ "Allocation of layer bandwidth and FEC for video multicast over wired and wireless networks," IEEE Trans. Circuits Syst. Video Technol., vol. 12, no. 12, pp. 1059-1070, Dec. 2002.

[23] I. Rhee, "Error control techniques for interactive low-bit rate video transmission over the Internet," in Proc. ACM SIGCOMM, 1998, pp. 290-301.

[24] T. Noguchi, M. Yamamoto, and H. Ikeda, "Reliable multicast protocol applied local EEC," in Proc. ICC 2001, vol. 8, 2001, pp. 2348-2353.

[25] R. Kermode, "Scoped hybrid automatic repeat request with forward error correction (SHAR.QFEC)," in Proc. ACM SIGCOMM, Sep. 1998, pp. 278-289.

[26] B. Li, "Reliable multicast transmissions using forward error correction and automatic retransmission requests," in Proc. Can. Conf. Electrical and Computer Engineering, vol. 2, 2001, pp. 1145-1150.

[27] J. Nonnenmacher, E. Biersack, and D. Towsley, "Parity-based loss recovery for reliable multicast retransmission," in Proc. ACM SIGCOMM, Sep. 1997.

[28] J. Nonnenmacher, E. W. Biersack, and D. Towsley, "Parity-based loss recovery for reliable multicast transmission," IEEE/ACM Trans. Networking, vol. 6, pp. 349-361, Aug. 1998.

[29] L. Xue and M. Ammar, "Bandwidth control for replicated-stream multicast video distribution," in Proc. 5th IEEE Int. Symp. High Performance Distributed Computing, Aug. 6-9, 1996, pp. 356-363.

[30] X. Zheng, S.-H. G. Chan, Q. Zhang, W.-W. Zhu, and Y.-Q. Zhang, "Feedback-free error recovery for video multicast," in Proc. IEEE ICC, May 11-15, 2003.

[31] B. Yan and K. W. Ng, "Mode-based error-resilient techniques for the robust communication of MPEG-4 video," IEEE Trans. Circuits Syst. Video Technol., vol. 14, no. 6, pp. 874-879, June 2004.

[32] J. Hagenauer and T. Stockhammer, "Channel coding and transmission aspects for wireless multimedia," Proc. IEEE, vol. 87, no. 10, pp. 1764-1777, Oct. 1999

[33] G.-H. Yang, D. Shen, and V. O. Li, "UEP for video transmission in spacetime coded OFDM systems," in Proc. IEEE Infocom, vol. 23, Mar. 2004, pp. 1201-1211.

[34] C. Dubuc, D. Boudreau, and F. Patenaude, "The design and simulated performance of a mobile video telephony application for satellite thirdgeneration wireless system," IEEE Trans. Multimedia, vol. 3, no. 4, pp. 424-431, Dec. 2001

[35] J. Kim, R. M. Mersereau, and Y. Altunbasak, "A multiple-substream unequal error-protection and error-concealment algorithm for SPIHTcoded video bitreams," in IEEE Trans. Image Process., vol. 13, Dec. 2004, pp. 1547-1553.

[36] G. Ren, H. Zhang, and Y. Chang, "A novel scheme for space-time block coding with a variable transmit diversity gain in OFDM systems," IEEE Trans. Consumer Electron., vol. 50, pp. 478-483, May 2004.
[37] T. Stockhammer, H. Jenkač, and C. Weiß, "Feedback and error protection strategies for wireless progressive video transmission," IEEE Trans. Circuits Syst. Video Technol., vol. 12, pp. 465-482, Jun. 2002.

[38] L. Xu, "Resource-efficient delivery of on-demand streaming using UEP codes," IEEE Trans. Commun., vol. 51, pp. 63-71, Jan. 2003.

[39] S. Kasera, G. Hjalmtusson, D. Towsley, and J. F. Kurose, "Scalable reliable multicast using multiple multicast channels," IEEE Trans. Networking, vol. 8, pp. 294-310, Jun. 2000.

[40] R. Prasad, C. Dovrolis, M. Murray, and K. Claffy, "Bandwidth estimation: Metrics, measurement techniques, and tools," IEEE Network, vol. 17, pp. 27-35, Nov.-Dec. 2003.

[41] S. Jongtae and R. Boorstyn, "Efficient loss estimation in high speed networks," in Proc. IEEE ATM Workshop, May 1998, pp. 360-367.

[42] R. Caceres, N. Duffield, J. Horowitz, D. Towlsey, and T. Bu, "Multicast-based inference of network-internal characteristics: Accuracy of packet loss estimation," in Proc. IEEE Infocom, vol. 1, Sept. 1999, pp. 371-379.

[43] Q. Zhang, W. Zhu, and Y.-Q. Zhang, "Channel-adaptive resource allocation for scalable video transmission over $3 \mathrm{G}$ wireless network," IEEE Trans. Circuits Syst. Video Technol., vol. 14, no. 8, pp. 1049-1063, Aug. 2004.

[44] A. Boyd, "Reports of Statistical Application Research (Union of Japanese Scientists and Engineers),", 1959. Inequalities for Mills' ratio.

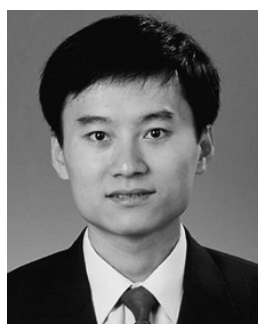

S.-H. Gary Chan (S'89-M'98-SM'03) received the B.S.E. degree (highest honor) in electrical engineering from Princeton University, Princeton, NJ, with certificates in applied and computational mathematics, engineering physics, and engineering and management systems, in 1993. He received the M.S.E. and Ph.D. degrees in electrical engineering from Stanford University, Stanford, CA, in 1994 and 1999, respectively, with a minor in business administration.

He is currently an Associate Professor with the Department of Computer Science, The Hong Kong University of Science and Technology, Hong Kong, and an Adjunct Researcher with the Microsoft Research Asia, Beijing, China. His research interest includes multimedia networking, peer-to-peer networks and wireless communications networks. He was a Visiting Assistant Professor in networking at the Department of Computer Science, University of California at Davis, from September 1998 to June 1999. During 1992-1993, he was a Research Intern at the NEC Research Institute, Princeton. He was a William and Leila Fellow at Stanford University during 1993-1994.

Dr. Chan currently serves as a Vice-Chair of IEEE COMSOC Multimedia Communications Technical Committee, and is a member of Tau Beta Pi, Sigma $\mathrm{Xi}$, and Phi Beta Kappa. At Princeton, he was the recipient of the Charles Ira Young Memorial Tablet and Medal, and the POEM Newport Award of Excellence in 1993.

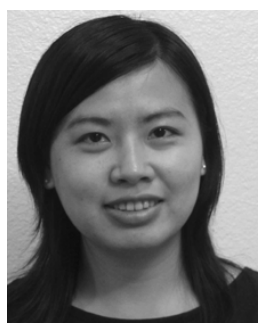

Xing Zheng received the B.E. in computer science and engineering from Zhejiang University, Hangzhou, China, and the M.Phil degree in computer science from the Hong Kong University of Science and Technology, Hong Kong. She is currently pursuing the Ph.D. degree from the School of Information and Computer Science, University of California, Irvine. Her research interests include sensor networks, distributed systems middleware, multimedia networking, image processing and analysis.

Ms. Zheng is a student member of the ACM. 


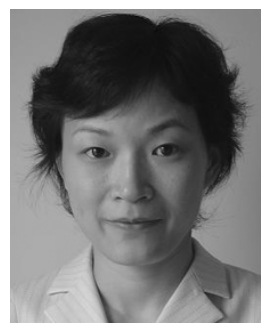

Qian Zhang (M'00-SM'04) received the B.S., M.S., and Ph.D. degrees from Wuhan University, China, in 1994, 1996, and 1999, respectively, all in computer science.

She joined Microsoft Research Asia, Beijing, China, in July 1999. She is currently the Research Manager of the Wireless and Networking group. She has published more than 80 refereed papers in international leading journals and key conferences in the arew of wireless/Internet multimedia networking, wireless communications and networking, and overlay networking. She is the inventor about 20 pending patents. Her current research interest includes seamless roaming across different wireless networks, multimedia delivery over wireless, Internet, next-generation wireless networks, and P2P network/ad hoc networks.

Dr. Zhang is a member of the Visual Signal Processing and Communication Technical Committee and the Multimedia System and Application Technical Committee of the IEEE Circuits and Systems Society. She is also a member and chair of QoSIG of the Multimedia Communication Technical Committee of the IEEE Communications Society. She is the Associate Editor for IEEE TRANSACTIONS ON VehICULAR TeChNOLOGIES. She is also serving as Guest Editor for a special issue on wireless video in IEEE Wireless Communication Magazine. She has also served in the technical committee of numerous IEEE and other international conferences. She has recently received TR, 100 (MIT Technology Review) world's top young innovator award.

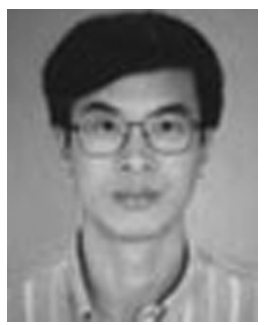

Wen-Wu Zhu (S'92-M'97-SM'01) received the B.E. and M.E. degrees from National University of Science and Technology of China (USTC), in 1985 and 1988, respectively, the M.S. degree from Illinois Institute of Technology, Chicago, and the Ph.D. degree from Polytechnic University, Brooklyn, New York, in 1993 and 1996, respectively, all in electrical engineering.

He joined Communication Technology Lab China as Co-Director in September 2004. Prior to his current post, he was with Microsoft Research Asia, Beijing, China, first as a Researcher in the Internet Media Group and later as Research Manager of Wireless and Networking Group. From 1996 to 1999, he was with Bell Labs, Lucent Technologies, NJ, as a Member of Technical Staff during 1996-1999. From 1988 to 1990, he was with the Graduate School, USTC, and the Institute of Electronics, Chinese Academy of Sciences, Beijing. He has published over 180 refereed papers in the areas of wireless/Internet multimedia delivery, and wireless communications and networking. He participated activity in the IETF ROHC WG on robust TCP/IP header compression over wireless links. $\mathrm{He}$ is co-inventor of over 20 pending patents. His current research interest is in the area of wireless communication and networking, and wireless/Internet multimedia communication and networking.

Dr. Zhu has been on various editorial boards of IEEE journals such as Guest Editor for the PROCEEDINGS OF THE IEEE, Associate Editor for the IEEE TRANSACTIONS ON MobILE COMPUTING, IEEE TRANSACTIONS ON Multimedia, IEEE TRANSACTIONS ON CiRcuits and Systems For VideO TECHNOLOGY (TCSVT). He received the Best Paper Award in IEEE TCSVT in 2001. He currently is also the Chairman of IEEE Circuits and System Society Beijing Chapter and the Secretary of Visual Signal Processing and Communication Technical Committee. He is a member of Eta Kappa Nu, Multimedia System and Application Technical Committee and Life Science Committee in IEEE Circuits and Systems Society, and Multimedia Communication Technical Committee in IEEE Communications Society.

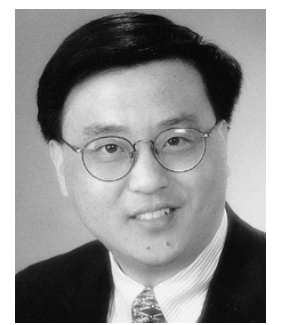

Ya-Qin Zhang (F'97) received the B.S. and M.S. degrees in electrical engineering from the University of Science and Technology of China (USTC) in 1983 and 1985, respectively, and the Ph.D. degree in electrical engineering from George Washington University, Washington, DC, in 1989. He had executive business training from Harvard University.

$\mathrm{He}$ is the Corporate Vice President of Microsoft Corporation in Redmond, Washington. He is currently responsible for product development of Microsoft's Mobile and Embedded Division, including WinCE operating system, Smartphone, PocketPC, and other Windows Mobile platform and devices. He was the Managing Director of Microsoft Research Asia, Microsoft's basic research arm in Asia-Pacific region. From 1994 to 1999. he was the Director of Multimedia Technology Laboratory at Sarnoff Corporation, Princeton, NJ (RCA Laboratories). He was with GTE (now Verizon) Corporation, Waltham, MA, from 1989 to 1994 . He has published over 300 refereed papers in leading international conferences and journals. $\mathrm{He}$ has been granted over 50 US patents in digital video, Internet, multimedia, wireless and satellite communications. Many of the technologies he and his team developed have become the basis for start-up ventures, commercial products, and international standards. He served on the Board of Directors of several IT companies, including Sohu.com (NASQ: SOHU)—a top Chinese portal.

Dr. Zhang served as the Editor-in-Chief for the IEEE TRANSACTIONS ON Circuits and Systems for Video Technology. He was the Chairman of Visual Signal Processing and Communications Technical Committee of IEEE Circuits and Systems. He serves on the Editorial boards of seven other professional journals and over a dozen conference committees. He has been a key contributor to the ISO/MPEG and ITU standardization efforts in digital video and multimedia. He received many awards, including several industry technical achievement awards and IEEE academic awards. 